\title{
Exploring the Dynamics of Triglyceride With Stroke Onset in the Chinese Population Through Parameterized Joint Models
}

\section{Qian Mao}

Weifang Medical University

\section{Wenfeng Gao}

Affiliated Hospital of Weifang Medical University

\section{Liping Yang}

Xijing Hospital of Fourth Military Medical University

Qian Zhao

Xijing Hospital of Fourth Military Medical University

Yujie Liu

Weifang Medical University

\section{Zhe Wang}

Weifang Medical University

\section{Peixia Guan}

Weifang Medical University

Huiwen Cong

Weifang Medical University

Lianyuan Wang

Weifang Medical University

\section{Yi Yang}

Weifang Medical University

\section{Gaopei Zhu}

Weifang Medical University

\section{Xiao Qi}

Weifang Medical University

\section{Yujia Kong}

Weifang Medical University

\section{Weijing Meng}

Weifang Medical University

Fuyan Shi ( $\nabla$ shifuyan@wfmc.edu.cn )

Weifang Medical University

\section{Suzhen Wang}


Weifang Medical University

\section{Research Article}

Keywords: Joint model, parameterization, triglyceride, stroke, longitudinal data

Posted Date: July 29th, 2021

DOI: https://doi.org/10.21203/rs.3.rs-709446/v1

License: (c) (1) This work is licensed under a Creative Commons Attribution 4.0 International License. Read Full License 


\section{Exploring the dynamics of triglyceride with stroke onset in}

\section{the Chinese population through parameterized joint models}

Qian $\mathrm{Mao}^{1 *}$, Wenfeng $\mathrm{Gao}^{2 *}$, Liping Yang ${ }^{3 *}$, Qian Zhao ${ }^{3 *}$, Yujie Liu ${ }^{1}$, Zhe Wang ${ }^{1}$, Peixia Guan ${ }^{1}$, Huiwen Cong ${ }^{1}$, Lianyuan Wang ${ }^{1}$, Yi Yang ${ }^{1}$, Gaopei Zhu ${ }^{1}$, Xiao Qi ${ }^{1}$, Yujia Kong ${ }^{1}$, Weijing Meng ${ }^{4}$, Fuyan Shi $^{{ }^{1}+}$, and Suzhen Wang ${ }^{1_{+}}$

${ }^{1}$ Department of Health Statistics, School of Public Health, Weifang Medical University, Weifang, Shandong, 261053, People's Republic of China

${ }^{2}$ Department of Immunology and Rheumatology, Affiliated Hospital of Weifang Medical University, Weifang, Shandong, 261053, People's Republic of China

${ }^{3}$ Health Medical Center, Xijing Hospital of Fourth Military Medical University, Xi'an, Shanxi, 710032, People's Republic of China

${ }^{4}$ School of Life Sciences and Technology, Weifang Medical University, Weifang, Shandong, 261053, People's Republic of China

* These authors contributed equally to this study and are co-first authors.

+ These authors are co-corresponding authors and are co-senior authors.

\section{Corresponding Author:}

Fuyan Shi, Suzhen Wang

Department of Health Statistics, School of Public Health, Weifang Medical University No.7166 Baotong West Street, Weifang, Shandong, People's Republic of China Email: shifuyan@wfmc.edu.cn(F.S.); wangsz@wfmc.edu.cn(S.W.) 


\title{
Exploring the dynamics of triglyceride with stroke onset in
}

\section{the Chinese population through parameterized joint models}

\begin{abstract}
Background: Stroke has become one of the diseases with the highest mortality and disability rates in the world, especially in low-income and developing countries. Our objective was to discuss the relationship between the longitudinal dynamic changes of TG and stroke onset in healthy population by constructing different parametric joint models.
\end{abstract}

Methods: 298 participants aged 23 to 69 in Xijing hospital of Xi'an City in Shanxi Province from 2008 to 2015 were included. The Cox proportional hazards model was performed to analyze the correlation between TG and stroke incidence at baseline. Different parameterized joint models were used to analyze the impact of dynamic changes of TG on the incidence of stroke under longitudinal data.

Results: Of the 298 participants, a total of 70 (23.49\%) subjects developed stroke during the study period. Cox proportional hazards model showed that the risk of disease increased by 1.056 times $(95 \% C I=0.920-0.975)$ for each 1 unit of baseline age decrease. Each $1 \mathrm{mmol} / \mathrm{L}$ increase in sqrt(TG) increased the risk by 1.816 times (95\%CI=1.017-3.245). Joint model showed that the risk of sqrt (TG) increased by 4.869 times $(95 \% C I=3.987-8.857)$ for each $1 \mathrm{mmol} / \mathrm{L}$ increase in longitudinal direction. The lagged effects $(H R=5.284,95 \% C I=4.397-9.680)$ and cumulative effects $(H R=1.786,95 \% C I=1.613-3.399)$ of sqrt $(\mathrm{TG})$ dynamic trajectory were also statistically related to the incidence of stroke. 
Conclusions: Over time, the longitudinal growth of TG levels in individuals will increase the risk of stroke even more. People should pay more attention to the dynamic changes of individual TG value, as well as the lagged effect and cumulative effect, to reduce the incidence of stroke.

Keywords: Joint model, parameterization, triglyceride, stroke, longitudinal data

\section{Background}

Stroke is a group of acute cerebrovascular diseases caused by a variety of pathogenic factors, leading to cerebrovascular block, short-term blood can not flow into the brain, or acute rupture of cerebral vessels caused by brain function damage [1]. Stroke is irreversible and difficult to treat. The high mortality, high disability rate and high recurrence rate caused by stroke will bring great disease burden to the society, which has become one of the major public health problems in the world. However, stroke can be also prevented effectively. Related research showed that $90.7 \%$ of global stroke was related to 10 correctable risk factors, such as hypertension, diabetes, dyslipidemia, smoking, drinking and abdominal obesity [2]. Therefore, primary prevention of stroke is the fundamental measure.

Some studies showed that there were correlation between the abnormal levels of TC, TG, LDL-C, HDL-C and the incidence of stroke [3,4]. And blood pressure, blood lipid, blood glucose, BMI and waist circumference are controllable factors. It will have a better guiding significance for individuals to formulate intervention measures 
if factors are changed, which can make the assessment objects more intuitively see the reduction degree of disease risk. However, at present, the research conclusions are not completely unified and need to be further explored.

In recent years, most studies focus on studying the relationship between the level of blood lipid indicators and the incidence of stroke through some traditional statistical models, such as logistic regression, Cox proportional hazards model, random forest and competitive risk model $[5,6]$. However, these studies lacked the consideration of the impact of the dynamic changes of longitudinal indicators, resulting in poor fitting effect and large error. To solve this problem, Faucett et al $[7,8]$. proposed a joint modeling method for longitudinal data and survival data, which could describe the dynamic change trajectory of longitudinal variables.

Joint model was first used in clinical trials of AIDS to evaluate and compare the efficacy of different drugs in the treatment of AIDS [9]. At present, it has been widely applied in many research fields [10-15], such as cancer, cardiovascular and cerebrovascular diseases, kidney disease, liver disease, heart surgery, mental health and other different diseases research. It has become a hot issue in the field of population health research, and provides a mature and powerful method to solve the longitudinal data research.

To our best knowledge, few studies have applied the combined joint model to explore the risk factors of stroke. Therefore, a longitudinal study based on the joint model was conducted to examine the influence of TG on stroke onset in our study, which can provide scientific basis for the effective prevention and control of stroke. 


\section{Methods}

\section{Data sources}

In this study, the longitudinal data of 298 subjects who had health examination from 2008 to 2015 in the health examination center of Xijing hospital in Xi'an City, Shanxi Province were used for analysis. The main variables included gender, date of birth, age, marital status, education level, height, weight, body mass index, blood pressure, fasting blood glucose, uric acid, TC, TG, and LDL-C, etc. All the study methods were performed in accordance with the Medical Ethics Committee of Weifang Medical University (NO.2021YX116). All the participants read and signed informed consent.

Quality control measures for anthropometric and laboratory measurements included the participants in the physical examination come from the same individual unit and all adopt the same test method. Before the physical examination, the subjects were required to be free of smoking, alcohol, coffee, and have a fasting stomach for more than 12 hours. Biochemical blood samples were collected 3-5ml from an antecubital vein of participants in the morning by the laboratory staff who had received unified training. Then, the samples were detected within 10-30 minutes after extraction. The unit of measurement of TG is $\mathrm{mmol} / \mathrm{L}$.

Inclusion criteria: (1) The number of physical examinations $\geq 3$ times; (2) There were no patients with diabetes, cardio-cerebrovascular disease, liver disease and kidney disease at baseline; (3) No missing baseline diagnosis information. Exclusion criteria: (1) The number of physical examinations $<3$ times; (2) Study subjects who 
already had stroke at baseline.

A total of 298 subjects were eventually included according to the inclusion and exclusion criteria of health check-up, 70 of whom developed stroke during the follow-up period. The diagnostic criteria of the end point and outcome variable (stroke) was international clinical diagnostic criteria.

\section{Statistical analysis}

In our study, we developed joint models to explore the association between longitudinal TG and stroke onset. The standard joint model was used to capture the relationship between TG and stroke, which can be generalized to the case of three parameterization models $[8,16,17]$. To better understand the lagged effects between the event and longitudinal parts in our model, we assumed that the risk of terminal event in time depended on the value of $\mathrm{TG}$ in the previous 3 years. Then, a time-dependent slopes parameterization joint model was also be postulated, in which the risk depended on both the current TG value of the trajectory and the slope of the true trajectory at time $t$. However, in many cases it may benefit by allowing the risk to depend on the longitudinal marker history, ie. the cumulative effect of TG.

In this study, we assumed $y_{i}(t)$ to be the follow-up measurements (TG) for patient $i(\mathrm{i}=1, \ldots, \mathrm{n})$ at time $t$, and we followed the framework of linear mixed effect model to fit the longitudinal outcomes [18]:

$$
\begin{gathered}
y_{i}(t)=m_{i}(t)+\varepsilon_{i}(t)=x_{i}^{T}(t) \beta+z_{i}^{T}(t) b_{i}+\varepsilon_{i}(t) \\
b_{i} \sim N(0, D), \varepsilon_{i}(t) \sim N\left(0, \sigma^{2}\right)
\end{gathered}
$$

Among them, $x_{i}^{T}(t)$ is the time-varying covariate with corresponding fixed effect 
term $\beta . z_{i}^{T}(t)$ is the time-varying covariate with corresponding fixed effect term $b_{i}$. According to the distribution requirements of longitudinal observed variables, square root transformation of TG index was required to meet the normal distribution [8].

For the survival submodel, we perform the Cox proportional hazards model [19]:

$$
h_{i}\left(^{t}\right)=h_{0}\left(^{t}\right)^{\exp }\left\{\gamma^{T} \omega_{i}+{ }^{\alpha} m_{i}\left(^{t}\right)\right\}
$$

Here, $h_{0}(t)$ donates the unspecified baseline risk function, $\gamma^{T}$ is the time-varying covariate with corresponding fixed effect term $\omega_{i}$, and the shared parameter $\alpha$ represents the impact of longitudinal results $m_{i}(t)$ on event risk.

The main parameter estimation method of the joint model was the maximum likelihood estimation method [20]. EM algorithm [21] or Newton Raphson algorithm [22] can be used to solve the maximum solution of log likelihood function. As a general iterative algorithm, EM algorithm is widely used.

In this study, quantitative data were calculated by means and standard deviation representation $(\bar{x} \pm s)$, and qualitative data were expressed both in frequencies and percentages, i.e.n(\%). Kaplan-Meier method was used for survival analysis, which was performed by survminer package of R 4.0.0 software. And the joint model was constructed by JM package of R 4.0.0 software. All tests were two-sided, and values of $P<0.05$ were considered statistically significant.

\section{Results}

\section{Descriptive analysis}

A total of 298 subjects were followed up and 1921 observational data were collected. Among them, the observation values of each research object include 
observation time, observation year, survival time, age, gender, TC, TG, HDL-C, LDL-C and outcome status etc. The dataset consisted of 204 males and 94 females, with a baseline age range of 23-69 years. The variable information was shown in Table 1.

The total population in this study was followed up for 3-8 years, with mean of follow-up time $6.48 \pm 1.54$ years. 288 people (96.64\%) were followed up for 4 years, 67 people $(82.89 \%)$ were followed up for 5 years, 215 people $(72.15 \%)$ were followed up for 6 years and 171 people (57.38\%) were followed up for 7 years. Only 106 subjects $(35.57 \%)$ were followed up for 8 years. A total of 70 subjects developed stroke during the study period, with an incidence density of 76.18 per 10,000 people per year. Among them, 58 were male and 12 were female, and the incidence density was 95.26 and 38.69 per 10,000 people per year, respectively. Figure. 1 showed the Kaplan-Meier plot of TG level and stroke cumulative incidence. The cumulative incidence of stroke was $23.49 \%$.

Figure. 1 Kaplan-Meier curve for cumulative incidence

Profile plot for TG biomarker were shown in Figure. 2. Figure.2 indicated that there were distinct differences in trajectories between those who had stroke during follow-up and those who did not have. And at each time point, the change trajectory of TG index had no obvious rule with observation time.

Figure. 2 Longitudinal trajectory plot of TG

\section{Results generated from Cox proportional hazards model}

Table 2 presented the association between incident stroke and $\operatorname{sqrt}(\mathrm{TG})$ at baseline. As can be seen, there were statistically significant differences in the 
influence of baseline age and $\operatorname{sqrt}(\mathrm{TG})$ on the incidence of stroke $(P<0.05)$. The risk of stroke increased by 1.056 -fold $(95 \% C I=0.920-0.975)$ for each 1 year decreased in baseline age. Similarly, the $H R$ for $\operatorname{sqrt}(\mathrm{TG})$ predicting stroke was 1.816 , which indicated that for every $1 \mathrm{mmol} / \mathrm{L}$ added to $\operatorname{sqrt}(\mathrm{TG})$ level for one subject, the increased risk for developing stroke was 1.816-fold $(95 \% C I=1.017-3.245)$.

\section{Results from standard joint model}

The results of standard joint model was shown in tables 3, which investigated the effect of dynamic change in sqrt(TG) on the hazard of stroke. In the longitudinal submodel, there was significant difference in the influence of gender on sqrt(TG) $(P<0.001)$, indicating that the average level of $\operatorname{sqrt}(\mathrm{TG})$ in males was 0.291 higher than that in females. The survival submodel in table 3 showed that there was a strong negative association between dynamic change in age and risk of stroke. A unit decrease in age represented as a 1.057 -fold $(95 \% C I=1.028-2.085)$ increase in developing stroke. Most importantly, the results also reflected a strong positive association between longitudinal augments in sqrt(TG) and the risk of developing stroke, which implied a unit longitudinal augment in sqrt(TG) represented a 4.869-fold (95\%CI=3.987-8.857) increase in the stroke.

\section{Results from parameterized joint models}

Three paramaterized joint models results were showed in table 4, with a significant negative association between dynamic change in age and risk of stroke in three submodels. However, the effect of age is not quite remarkable in time-dependent slopes parameterization joint model $(P>0.05)$. Additionally, shared parameters $\alpha$ 
were statistically significant in the lagged effect joint model $(P<0.001)$, which demonstrated that one unit longitudinal increase in $\operatorname{sqrt}(\mathrm{TG})$ level three years earlier was associated with a 5.284-fold $(95 \% C I=4.397-9.680)$ increase in the risk of developing the disease. Similarly to the above analyses, we observed that sqrt(TG) remains strongly related with the risk for stroke $(P<0.001)$. In particular, a unit increase in the area under the sqrt(TG) longitudinal profile corresponds to a 1.786-fold $(95 \% C I=1.613-3.399)$ increase in the risk for stroke onset. In the time-dependent slopes parameterization joint model submodel, although the slope of the trajectory was not strongly associated with onset $(P>0.05)$, a unit longitudinal increase in sqrt(TG) still increased the risk of the onset $(P<0.001)$.

\section{Discussion}

Stroke incidence rate is the second leading cause of death and disability in the world. In recent years, the incidence of stroke has been increasing worldwide. However, the risk factors for stroke are still not clear [23]. At present, age, gender, hypertension, diabetes, dyslipidemia, heart disease, unhealthy diet and other factors are recognized as the main risk factors of stroke, and different risk factors have different effects on stroke. In this study, we conducted a preliminary analysis of the association between longitudinal $\mathrm{TG}$ trajectories and stroke onset. Although dyslipidemia as a risk factor for stroke has been controversial, more and more epidemiological evidence supports the role of dyslipidemia in increasing the risk of stroke and represents a potential target for therapeutic intervention [24]. Therefore, we hope that this study can provide a methodological basis for the exploration of the 
etiology of such chronic diseases.

In the Cox proportional hazards model, we found that low age and high TG levels at baseline were associated with the risk of stroke, which was not completely consistent with other research results. Mi $\mathrm{T}$ et al [25]. indicated that $\mathrm{TG}$ was an independent risk factor for carotid plaque, thus identifying a high risk of stroke. This finding was consistent with other previous studies [26]. However, Gainey J et al [27]. found that old age was one of the risk factors of stroke through Cox regression analysis. The inconsistent finding may be related to the rapid rise and younger incidence of stroke in China. The average age of stroke in China was about 65 years old, lower than that in developed countries, about 75 years old [28]. In fact, due to the relative improvement of risk awareness in the elderly from China, combined with lifestyle changes and lipid-lowering drugs, the risk of dyslipidemia and the incidence of cardiovascular and cerebrovascular diseases can be reduced to a certain extent. On the contrary, more and more young people were attacked by cardiovascular and cerebrovascular diseases because of drinking, smoking, excessive night life, high-fat diet, hypertension and other risk factors.

In addition, there were many reasons for inconsistent results. The traditional statistical model did not consider the dynamic changes of individual indicators over time and the influence of other covariates, which may lead to some biases in the results. In this study, we used the joint model to dynamically analyze the association between TG and stroke incidence, considering the influence of dynamic changes of index on disease progression, so as to more accurately analyze the rule of index 
values changing over time from the perspective of individuals. Our findings demonstrated that there was a strong positive association between longitudinal augments in sqrt(TG) and the risk of stroke onset. More importantly, the dynamic increase of TG level in individuals had a greater impact on stroke than at baseline, which may be due to the simultaneous consideration of baseline and longitudinal dynamic changes of indicator. This result was the most significant feature of the present study and corresponded with our purpose. It was consistent that Lee $\mathrm{j}$ et al [29]. in a 17.7-year prospective cohort study in the United States found that adults with high TG had a higher risk of stroke $(H R=1.32,95 \% C I=1.06-1.64)$. Interestingly, Wang $\mathrm{Y}$ [30] stratified the primary prevention population of stroke in China, and compared the correlation between the blood lipid level and the incidence of stroke among different levels. He believed that the incidence of stroke increased with the increase of age and TG level, and the correlation between the risk of stroke and serum TG level was greater in the population with the highest age, which was an inconclusive one from our research. In consequence, the relationship between age and stroke onset needed to be further explored.

Our study also constructed other different parameterized joint models for longitudinal data, to further analyze the correlation between different parameterization longitudinal marker sqrt(TG) and stroke onset. And we concluded that the longitudinal observation sqrt(TG) had a time lag effect and cumulative effect on the disease outcome. This was unexpected and novel, as other studies had found lagged associations between some climatic factors, environmental factors as well as social 
factors and stroke onset [31-33]. The lag effect of dyslipidemia on stroke needed to be further explored. However, this study can provide new ideas for the etiological inference and prognosis of such diseases. Similarly, the cumulative effect joint model can also provide a feasible parameterization method for the construction of the etiology exploration model of stroke to a certain extent. Because, so far, a common feature of the parameterization we had seen was that they assumed the risk of a specific time event depended only on the longitudinal trajectory characteristics of a single point in time. However, some scholars believed that this hypothesis was not always true. In many cases, we can benefit from the more detailed function of allowing the risk of an event to depend on the longitudinal observation history $[34,35]$.

We had expected that the slope term of $\operatorname{sqrt}(\mathrm{TG})$ longitudinal trajectory would have an effect on the outcome of the disease. However, we failed to find the association between them, and only the real level of $\operatorname{sqrt}(\mathrm{TG})$ at the same time point on the risk of stroke was found. The reason may be that the TG of the human body changed with the function of the immune system. Nevertheless, up to now, the time-dependent slopes parameterization joint model had been mostly used in the study of the relationship between biomarkers and risk of chronic diseases, used to capture the trajectory slope of observation indicators and the degree of risk [36]. In the future study, it is necessary to determine the specific applicable diseases and indicators of this kind of parametric joint model, as well as the parameter setting in the modeling process. 
Nevertheless, this study also had some limitations. First, due to the limitation of data collection, the influence of TG observation value on the stroke onset was only analyzed, instead of considering comprehensive factors, such as inflammatory factors, living habits, occupation, marriage, education level and so on. In the future study, more covariates should be considered to infer the risk factors of stroke onset. Second, the incidence of stroke was the result of long-term dynamic development, so the follow-up period of this study was still limited, which may lead to the deviation of model fitting. Third, the joint model established in this study can only deal with single longitudinal marker. The related researches showed that multiple longitudinal results modeled simultaneously, which can enhance model prediction ability and facilitate the guidance model for predicting stroke onset risk [37]. Therefore, it can be extended to the multivariable joint model dealing with multiple longitudinal markers in future research.

\section{Conclusions}

The joint model can be used to better understand the disease progression by analyzing longitudinal monitoring variables. In this study, we found that the longitudinal increase of individual TG level over time would increase the risk of stroke. People should pay close attention to the change trend of TG and various other blood lipid indicators, and the growth of indicators in the normal range can not be ignored, while maintaining a healthy lifestyle, such as healthy diet and active exercise. In addition, we found that the true longitudinal trajectory of TG, the lagged effect and cumulative effect three years ago were all strongly correlated with the stroke onset. In 
the future research of stroke etiology, we should pay more attention to the lagged effect and cumulative effect of TG. Future study should be further developed to clarify how the slope of TG locus affects the incidence of stroke.

\section{Abbreviations}

TG: Triglyceride. HR: Hazard Ratio. CI: Confidence Interval. TC: Total cholesterol. HDL-C:

High density lipoprotein cholesterol. LDL-C: Low density lipoprotein cholesterol. BMI: Body mass index. AIDS: Acquired immunodeficiency syndrome. EM: Expectation Maximization.

\section{Declarations}

\section{Ethics approval and consent to participate}

The study was approved by Medical Ethics Committee of Weifang Medical University (NO.2021YX116). All the methods were performed in accordance with the Medical Ethics Committee. All the participants read and signed informed consent.

\section{Consent for publication}

All authors have approved the manuscript for submission.

\section{Availability of data and materials}

The datasets generated and analysed during the current study are not publicly available due to medical ethics but are available from the corresponding author on reasonable request.

\section{Competing interests}

The authors declare that they have no competing interests

\section{Funding}


The authors disclosed receipt of the following financial support for the research, authorship, and/or publication of this article: This work was funded by the National Natural Science Foundation of China (Project approval No.81803337, 81872719), the Shandong Provincial Youth Innovation Team Development Plan of Colleges and Universities (No.2019-6-156), the National Bureau of Statistics Foundation Project (Project approval No.2018LY79), the Natural Science Foundation of Shandong Province (Project approval No.ZR2019MH034), and the Doctor Start-up Fund of Weifang Medical University (2017BSQD51). The funders did not play any role in the study design, collection and interpretation of data, or in writing the manuscript.

\section{Authors' contributions}

QM and WG made equal contributions to the structural design and manuscript writing. LY and QZ provided the longitudinal data collection from Xijing hospital of Xi'an City in Shanxi Province of China, and the basic data analysis. FS and SW adjusted the modeling program and reviewed the manuscript. YL, ZW and PG discussed and performed the modeling analysis codes. HC, LW and YY made contributions to the data collection and consolidation. GZ, XQ, YK and WM reviewed the structural design and writing problems. All authors read and approved the final manuscript.

\section{Acknowledgements}

The authors acknowledge the valuable support of the data provided by the Xijing hospital of Xi'an City in Shanxi Province of China and individuals who take part in this study and share their knowledge and attitudes.

\section{Authors' information}

${ }^{1}$ Department of Health Statistics, School of Public Health, Weifang Medical University, Weifang, Shandong, 261053, People's Republic of China. ${ }^{2}$ Department of Immunology and Rheumatology, 
Affiliated Hospital of Weifang Medical University, Weifang, Shandong, 261053, People's Republic of China. ${ }^{3}$ Health Medical Center, Xijing Hospital of Fourth Military Medical University, Xi'an, Shanxi, 710032, People's Republic of China. ${ }^{4}$ School of Life Sciences and Technology, Weifang Medical University, Weifang, Shandong, 261053, People’s Republic of China.

\section{References}

1. Kim J, Gall SL, Dewey HM, Macdone11 RA, Sturm JW, Thrift AG. Baseline Smoking Status and the Long-Term Risk of Death or Nonfatal Vascular Event in People with Stroke A 10-Year Survival Analysis. Stroke; a journal of cerebral circulation. 2012; 43(12): 3173. https://doi. org/10. 1161/STROKEAHA. 112. 668905

2. 0’ Donne11 MJ, Chin SL, Rangarajan S, Xavier D, Liu L, Zhang H, et al. Global and regional effects of potentially modifiable risk factors associated with acute stroke in 32 countries (INTERSTROKE) : a case-control study. Lancet. 2016;388 (10046) :761-75. https://doi. org/10.1016/S0140-6736(16) 30506-2.

3. Lee JH, Park SH, Yang DH, Park HS, Cho Y, Lee WK, et al. Threshold level of low-density lipoprotein cholesterol for the short-term benefit of statin therapy in the acute phase of myocardial infarction. Clin Cardiol. 2012;35(4):211-8. https://doi. org/10. 1002/c1c. 20984.

4. Wieberdink RG, Poels MM, Vernooij MW, Koudstaal PJ, Hofman A, van der Lugt A, et al. Serum lipid levels and the risk of intracerebral hemorrhage: the Rotterdam Study. Arterioscler Thromb Vasc Biol. 2011;31(12):2982-9.

https://doi. org/10. 1161/ATVBAHA. 111. 234948. 
5. Guo X, Li Z, Sun G, Guo L, Zheng L, Yu S, et al. Comparison of four nontraditional lipid profiles in relation to ischemic stroke among hypertensive Chinese population. Int J Cardiol. 2015;201:123-5. https://doi. org/10. 1016/j. ijcard.

6. de la Riva P, Zubikarai M, Sarasqueta C, Tainta M, Muñoz-Lopetegui A, Andr és-Marín N, et al. Nontraditional Lipid Variables Predict Recurrent Brain Ischemia in Embolic Stroke of Undetermined Source. J Stroke Cerebrovasc Dis. 2017;26 (8):1670-1677. https://doi.org/10.1016/j. jstrokecerebrovasdis.

7. Faucett CL, Thomas DC. Simultaneously modelling censored survival data and repeatedly measured covariates: a Gibbs sampling approach. Stat Med. 1996;15(15) : 1663-85. https://doi. org/10. 1002/(SICI) 1097-0258(19960815) 15: 15<1663: :AID-SIM294>3. 0. C0;2-1.

8. Rizopoulos D. Joint Models for Longitudinal and Time-to-Event Data: With Applications in R (1st ed.).Chapman and Hall/CRC; 2012. https://doi. org/10.1201/b12208.

9. Tsiatis AA, Degruttola Victor, Wulfsohn MS. Modeling the Relationship of Survival to Longitudinal Data Measured with Error. Applications to Survival and CD4 Counts in Patients with AIDS. Publications of the American Statistical Association. 1995;90(429): 27-37.

10. Andrinopoulou ER, Rizopoulos D, Takkenberg JJ, Lesaffre E. Combined dynamic predictions using joint models of two longitudinal outcomes and competing risk data. Stat Methods Med Res. 2017;26(4):1787-1801. 
https://doi. org/10. 1177/0962280215588340.

11. Sweeting MJ, Thompson SG. Joint modelling of longitudinal and time-to-event data with application to predicting abdominal aortic aneurysm growth and rupture. Biometrical Journal. 2011;53(5):750-763.

https://doi. org/10. 1002/bimj. 201100052.

12. Wang C, Douglas J, Anderson S. Item response models for joint analysis of quality of life and survival. Stat Med. 2002;21:129-142.

https://doi. org/10. 1002/sim. 989.

13. Dejardin D, Lesaffre E, Verbeke G. Joint modeling of progression-free survival and death in advanced cancer clinical trials. Statistics in Medicine. 2010;29(16): 1724-1734. https://doi. org/10. 1002/sim. 3918.

14. Terrin N, Rodday AM, Parsons SK. Joint models for predicting transplant-related mortality from quality of life data. Qual Life Res. 2015;24(1):31-39. https://doi. org/10. 1007/s11136-013-0550-2.

15. Yang L, Yu M, Gao S. Prediction of coronary artery disease risk based on multiple longitudinal biomarkers. Stat Med. 2016;35: 1299-1314. https://doi. org/10. 1002/sim. 6754.

16. Cavender JB, Rogers WJ, Fisher LD, Gersh BJ, Coggin CJ, Myers W0. Effects of smoking on survival and morbidity in patients randomized to medical or surgical therapy in the Coronary Artery Surgery Study (CASS): 10-year follow-up. CASS Investigators. J Am Coll Cardiol. 1992;20(2) :287-94. https://doi. org/10. 1016/0735-1097(92) 90092-2. 
17. Hauptmann M, Richardson DB. Flexible modeling of the cumulative effects of time-dependent exposures on the hazard. Stat Med. 2011;30(2):197; author reply 198-9. https://doi. org/10. 1002/sim. 4007.

18. Van der Elst W, Molenberghs G, Hilgers RD, Verbeke G, Heussen N. Estimating the reliability of repeatedly measured endpoints based on linear mixed-effects models. A tutorial. Pharm Stat. 2016;15(6) :486-493. https://doi. org/10. 1002/pst. 1787.

19. Cox DR. Regression Mode1s and Life-Tables. Journal of the royal statistical society series b-methodological. 1972;34(2): 187-220.

20. Schuler MS, Rose S. Targeted Maximum Likelihood Estimation for Causal Inference in Observational Studies. Am J Epidemiol. 2017;185(1):65-73. https://doi. org/10. 1093/aje/kww165.

21. Ratcliffe SJ, Guo W, Ten Have TR. Joint modeling of longitudinal and survival data via a common frailty. Biometrics. 2004;60(4):892-9. https://doi. org/10.1111/j. 0006-341X. 2004.00244.x.

22. Hickey GL, Philipson P, Jorgensen A, Kolamunnage-Dona R. joineRML: a joint model and software package for time-to-event and multivariate longitudinal outcomes. BMC Medical Research Methodology. 2018;18(1):50. https://doi. org/10. 1186/s12874-018-0502-1.

23. Wang W, Jiang B, Sun H, Ru X, Sun D, Wang L, et al. Prevalence, Incidence, and Mortality of Stroke in China: Results from a Nationwide Population-Based Survey of 480687 Adults. Circulation. 2017;135(8):759-771. 
https://doi. org/10. 1161/CIRCULATIONAHA. 116. 025250.

24. Barkas F, Milionis H. Treating Dyslipidemia for the Primary and Secondary Prevention of Stroke. Semin Neurol. 2017;37 (3):286-293.

https://doi. org/10. 1055/s-0037-1603511.

25. Mi T, Sun S, Zhang G, Carora Y, Du Y, Guo S, et al. Relationship between dyslipidemia and carotid plaques in a high-stroke-risk population in Shandong Province, China. Brain Behav. 2016;6(6) : e00473.

https://doi. org/10. 1002/brb3. 473.

26. Djelilovic-Vranic J, Alajbegovic A, Zelija-Asimi V, Niksic M, Tiric-Campara M, Salcic S, et al. Predilection role diabetes mellitus and dyslipidemia in the onset of ischemic stroke. Med Arch. 2013;67(2):120-3.

https://doi. org/10. 5455/medarh. 2013.67. 120-123.

27. Gainey J, Blum B, Bowie B, Cooley K, Madeline L, Ervin EL, et al. Stroke and dyslipidemia: clinical risk factors in the telestroke versus non-telestroke. Lipids Health Dis. 2018;17(1):226.

https://doi. org/10. 1186/s12944-018-0870-x.

28. Li S, Zeng M, Dong J, Li M, Yan X, Li R, et al. Management of Endovascular Treatment for Acute Ischemic Stroke During the COVID-19 Pandemic at a Single Institution in Beijing, China: A Brief Report. J Neurosurg Anesthesiol. 2021;33 (3) :268-272. https://doi. org/10. 1097/ANA. 0000000000000748.

29. Lee JS, Chang PY, Zhang Y, Kizer JR, Best LG, Howard BV. Triglyceride and HDL-C Dyslipidemia and Risks of Coronary Heart Disease and Ischemic Stroke 
by Glycemic Dysregulation Status: The Strong Heart Study. Diabetes Care. 2017; 40 (4):529-537. https://doi. org/10. 2337/dc16-1958.

30. Wang Y. The relationship for blood lipid levels and stroke incidence in Chinese rural population aged $45^{\sim} 75$ with primary hypertension. Fudan University; 2014.

31. Shah AS, Lee KK, McAllister DA, Hunter A, Nair H, Whiteley W, et al. Short term exposure to air pollution and stroke: systematic review and meta-analysis. BMJ. 2015;350:h1295. https://doi. org/10.1136/bmj.h1295.

32. Luo Y, Li H, Huang F, Van Halm-Lutterodt N, Qin Xu, Wang A, et al. The cold effect of ambient temperature on ischemic and hemorrhagic stroke hospital admissions: A large database study in Beijing, China between years 2013 and 2014-Utilizing a distributed lag non-linear analysis. Environ Pollut. 2018;232:90-96. https://doi. org/10. 1016/j. envpo1. 2017. 09. 021.

33. Lian H, Ding X, Zhang H, Wang X. Short-term effect of stock volatility and cardiovascular mortality: a systematic review and meta-analysis. Ann Trans1 Med. 2020; 8(20):1317. https://doi. org/10.21037/atm-20-6557.

34. Hauptmann M, Wellmann J, Lubin JH, Rosenberg PS, Kreienbrock L. Analysis of exposure-time-response relationships using a spline weight function. Biometrics. 2000; 56(4):1105-8. https://doi. org/10.1111/j. 0006-341x. 2000.01105.x.

35. Sylvestre MP, Abrahamowicz M. Flexible modeling of the cumulative effects of time-dependent exposures on the hazard. Stat Med. 2009; $28(27): 3437-53$. 
https://doi. org/10. 1002/sim. 4007.

36. Li K, Furr-Stimming E, Paulsen JS, Luo S; PREDICT-HD Investigators of the Huntington Study Group. Dynamic Prediction of Motor Diagnosis in Huntington's Disease Using a Joint Modeling Approach. Journal of Huntington's Disease. 2017; 6(2):127-137.

https://doi. org/10. 3233/JHD-170236.

37. Andrinopoulou ER, Clancy JP, Szczesniak RD. Multivariate joint modeling to identify markers of growth and lung function decline that predict cystic fibrosis pulmonary exacerbation onset. BMC Pulm Med. 2020; 20(1):142. https://doi. org/10. 1186/s12890-020-1177-z. 
Figures
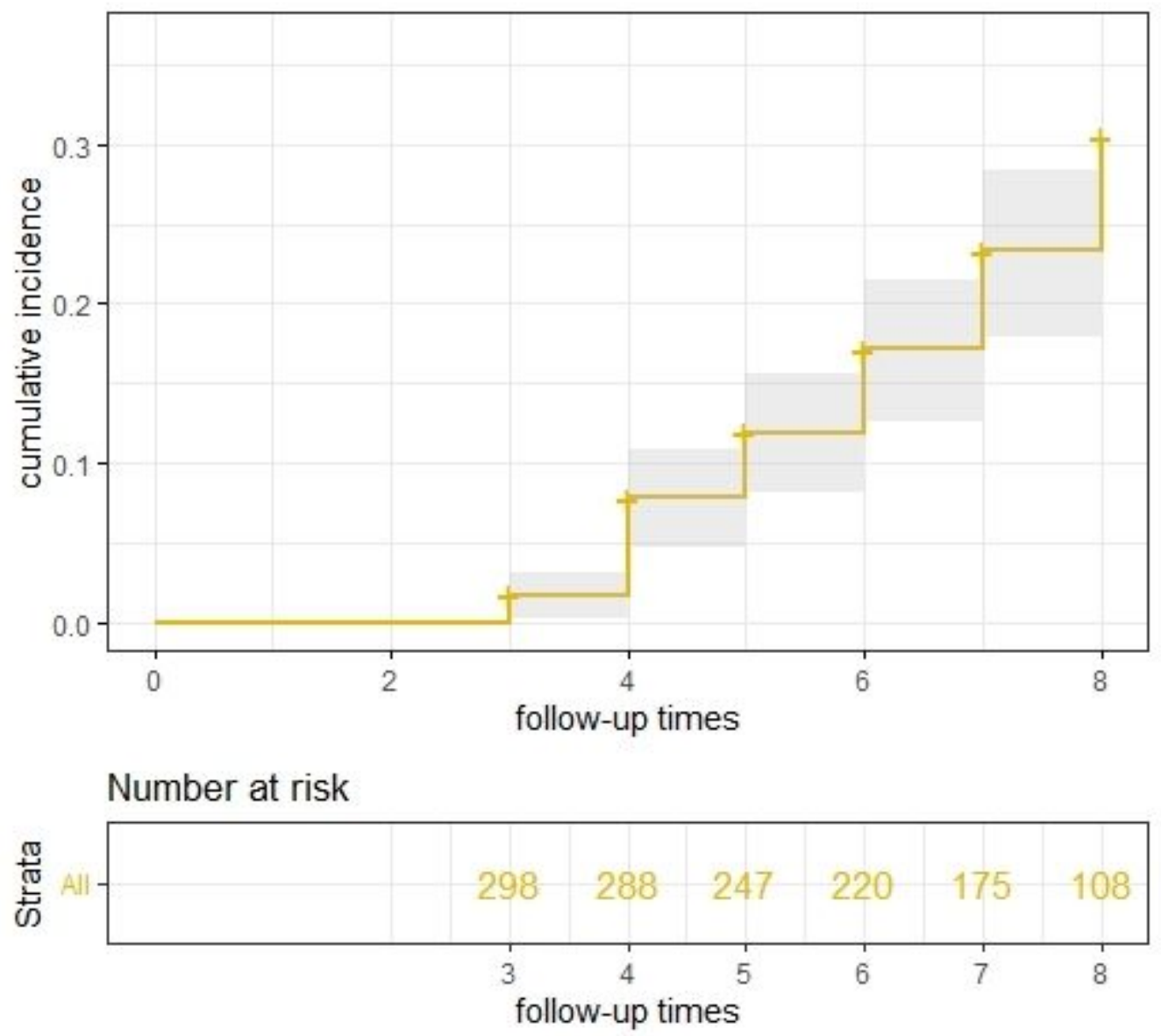

Figure 1

Kaplan-Meier curve for cumulative incidence 


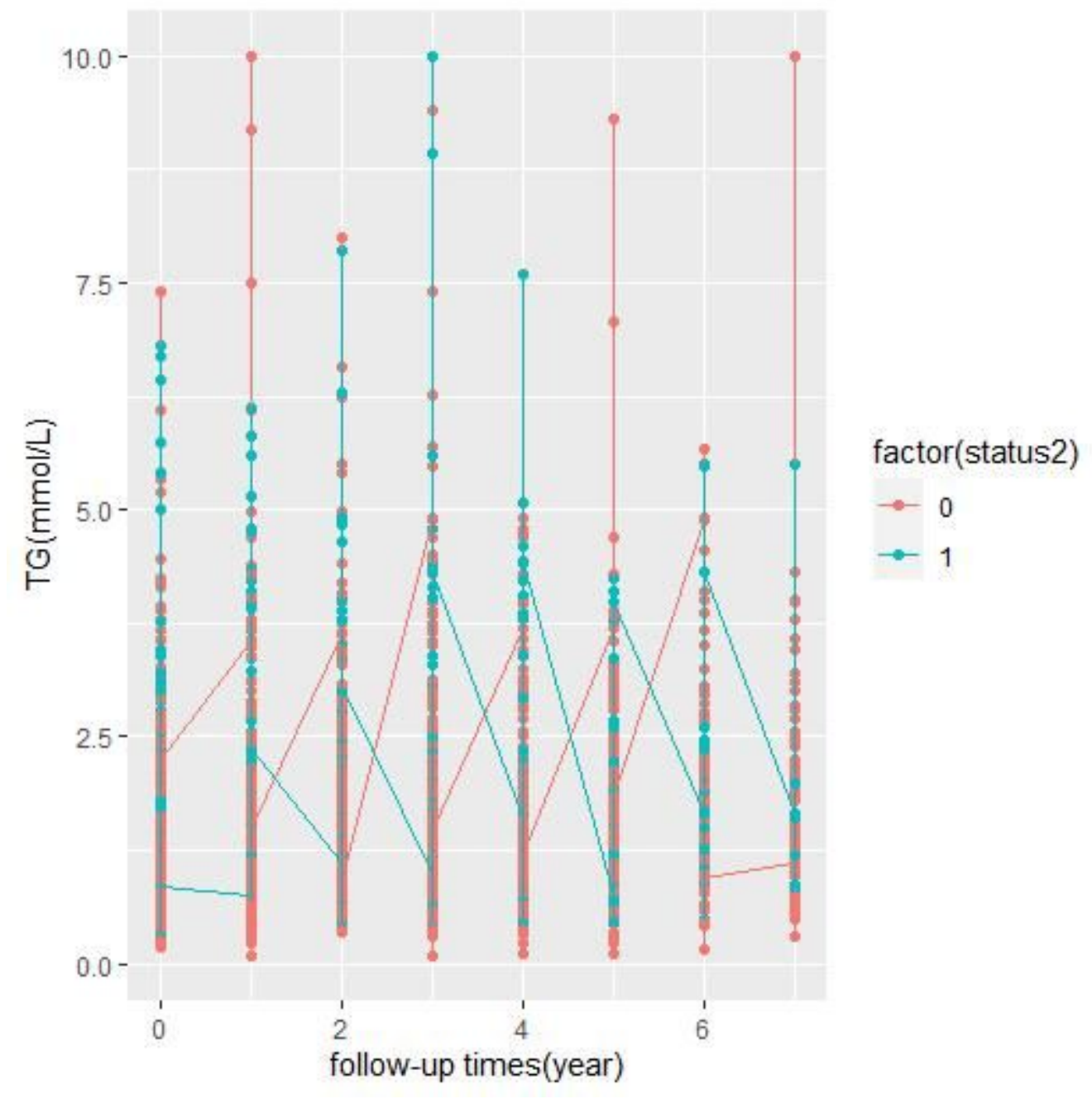

Figure 2

Longitudinal trajectory plot of TG 